vacuum ( $10^{-6}$ Torr $)$ with a high power electric field. This caused electrothermal self-heating of the GNR to hundreds of degrees which removed $p$-doping sources. The researchers, then, functionalized GNRs by introducing electron-rich nitrogen species by e-annealing the GNR devices in a $\sim 1$-Torr $\mathrm{NH}_{3} /$ Ar environment. The GNR devices functionalized in this way showed Dirac point positions shifted by $\sim-20 \mathrm{~V}$ before and after e-annealing in $\mathrm{NH}_{3}$, that stayed stable and constant in vacuum, signifying $n$-type electronic dop- ing without degradation of their carrier mobility. The researchers demonstrated by $\mathrm{x}$-ray photoelectron spectroscopy and nanometer-scale secondary ion mass spectroscopy that the thermal annealing with $\mathrm{NH}_{3}$ generated carbon-nitrogen species mostly at the edges of the graphene where chemical reactivity is high. Theoretical calculations performed by the researchers showed that GNRs functionalized by oxygen and nitrogen species on their edges were $p$ - and $n$-doped, respectively, which agreed with the experimental results the researchers obtained.

Using the e-annealing approach in $\mathrm{NH}_{3}$, the researchers fabricated $n$-type sub- $10 \mathrm{~nm}$ GNRFETs with Ti contact metals and a $5 \mathrm{~nm}$ Pd buffer layer that operated at room temperature with a subthreshold slope similar to that of the as-made $p$-type GNRFETs. The researchers said that the ability to control graphene chemistry through edge doping at the nanoscale is an important step toward controlled graphene electronics.

JOAN J. CARVAJAL

\section{Light-Assisted Writing of Bits Achieved on Low-Doped (Ga,Mn)As Ferromagnetic Semiconductors}

Energy-assisted switching mechanisms for magnetic bits will be needed in future magnetic recording technology. These mechanisms use a second source of energy to reduce the material's coercivity, the applied magnetic field needed to reverse the orientation of the magnetization of a ferromagnet. Mechanisms under consideration include heating a magnetic material with a laser or stimulating it with a transverse microwave field. Ideally, such a method should be local and reversible: It should reduce the coercivity of a bit only where it is applied and only for so long as it is applied, after which it should recover fully. G.V. Astakhov and coworkers of the University of Würzburg, Germany and V.L. Korenev of the Russian Academy of Sciences have recently found just such a mechanism in low-doped (Ga,Mn)As thin films at low temperatures using the photocoercivity effect. By removing the need for heating, this could provide a low power alternative to other energy-assisted recording technologies.

The researchers demonstrated lightassisted writing in the May 8 issue of Physical Review Letters (DOI: 10.1103/ PhysRevLett.102.187401; \#187401). They used low-temperature molecular beam epitaxy to deposit a $360 \mathrm{~nm}$ layer of $\left(\mathrm{Ga}_{1-x} \mathrm{Mn}_{x}\right)$ As ferromagnetic semiconductors, with $x \approx 0.005$. These samples were measured at $2 \mathrm{~K}$ with the magnetooptical Kerr effect at two different laser powers, the "dark" condition at $10 \mu \mathrm{W}$ and the "light" condition at $1 \mathrm{~mW}$. Finding the coercivity to be 525 Oe and 285 Oe, respectively, the researchers returned to $10 \mu \mathrm{W}$ and found the coercivity recovered to its original value-showing that the effect is reversible. They then wrote patterns on the substrate in an intermediate field of $470 \mathrm{Oe}$, evidencing both the locality of the transition and its potential to write bits.

The mechanism the researchers propose for this behavior is the mobility of holes. The magnetic behavior of the (Ga,Mn)As is mediated by the holes, and in low-doped samples, the mobility is low. This means that the holes are not free to relocate to their most favored state when a field is applied, and therefore some extra field is required to move domain walls. In comparison, the photocoercivity effect was not seen in more highly doped samples with $x \approx 0.05$, in which holes move readily. This mechanism provides a clue for materials engineers on how to extend this effect to low-power light-assisted magnetic recording in consumer products.

JIM RANTSCHLER

\section{LaSrCoFeO Offers Alternate Cathode Material to Pt for Micro-Fuel Cells}

With the proliferation of portable electronic devices, there is a critical need for reliable power sources for these devices. One solution to meet this increasing demand is the use of fuel cells. In particular, solid oxide fuel cells (SOFCs) are relatively very efficient and are flexible in the type of fuel they can use. However, SOFCs typically operate above $700^{\circ} \mathrm{C}$. It would be advantageous for them to operate at lower temperatures in the $200-600^{\circ} \mathrm{C}$ range, allowing for greater material flexibility and efficient operation as well as reduced corrosion than for current systems. SOFCs typically use yttriastabilized zirconia (YSZ) electrolyte. Researchers have demonstrated $\mathrm{Pt}$ cathodes for operation of YSZ-based SOFCs at intermediate temperatures. However, the
Pt may degrade at the higher temperatures in this range, and materials such as $\mathrm{La}_{0.6} \mathrm{Sr}_{0.4} \mathrm{Co}_{0.8} \mathrm{Fe}_{0.2} \mathrm{O}_{3-\delta}$ (LCSF) have been proposed and tested as alternatives to $\mathrm{Pt}$ for intermediate temperature SOFC cathodes. In a recently reported study, A.C. Johnson, B.-K. Lai, H. Xiong, and S. Ramanathan of Harvard University describe the fabrication of micro-SOFCs using dense ultrathin-film LSCF cathodes, formed using RF-sputtering, and YSZ electrolyte films. The results of their study were published in the January issue of the Journal of Power Sources (DOI: 10.1016/ j.jpowsour.2008.10.021; p. 252).

The thin-film SOFCs were fabricated with both $\mathrm{Pt}$ and mixed conducting oxide cathodes using sputtering, lithography, and etching. Each device is comprised of 75-150-nm thick YSZ electrolyte, a 40-80-nm porous $\mathrm{Pt}$ anode, and a cathode made of 15-150-nm dense LSCF or 130-nm porous
Pt. Several devices were fabricated to systematically investigate the electrical properties of the individual components of these fuel cells. For fuel cell measurements, five fuel cells were developed including three LSCF/YSZ/Pt cells (LCSF cathode) and two Pt/YSZ/Pt cells (Pt cathode). While the Pt-cathode cells produced the greatest power at most temperatures, the LCSF-cathode cells were not far behind. For instance, at $500^{\circ} \mathrm{C}$, power densities of $90 \mathrm{~mW} \mathrm{~cm}^{-2}$ and $60 \mathrm{~mW} \mathrm{~cm}^{-2}$ were observed for the Pt and LSCF cathodes, respectively. The latter is comparable to previous reports using ultrathin-film oxides.

The major advantage of the present approach is that the thin-film cathode processing is compatible with current photolithography and patterning. It is relatively simple to synthesize a multicomponent, highly dense complex oxide thin film from a single target. The results suggest that the 
use of non-precious metal cathodes for low- and intermediate-temperature thinfilm SOFCs is indeed feasible. The results are of major relevance to microscale energy conversion devices for portable appli- cations, and future devices will likely use similar SOFCs as power sources. Also, very recently, the researchers reported low-temperature electrochemical characterization of the ultrathin LSCF cathodes and have obtained mechanistic insights into their performance in micro-fuel cells (Journal of Power Sources, DOI: 10.1016/ j.jpowsour.2009.04.024).

GOPAL RAO

\section{News of MRS Members/Materials Researchers}

\section{George M. Whitesides to Receive Inaugural Dreyfus Prize in the Chemical Sciences}

George M. Whitesides, the Woodford L. and Ann A. Flowers University Professor of Chemistry at Harvard University, has been

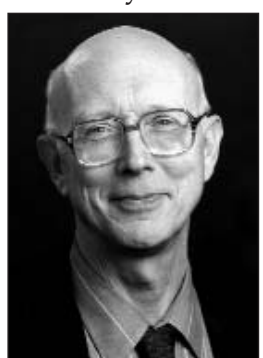

George M. Whitesides named to receive the inaugural Dreyfus Prize in the Chemical Sciences for revolutionizing the chemistry of soft materials.

The prize, to be given biennially by the Camille and Henry Dreyfus Foundation, recognizes exceptional and original research in a selected area of chemistry that has advanced the field in major ways. Conferred this year in materials chemistry, the prize consists of a monetary award of $\$ 250,000$, a citation, and a medal. The award ceremony will be at Harvard University on September 30, and will include a lecture by Whitesides.

Whitesides has developed powerful methods for the creation of new materials that have significantly advanced the field of chemistry and its societal benefits. His research extends across multiple disciplines, centered on chemistry, but touching biochemistry, drug design, and materials science. His work extends to the engineering of functional systems and the applications of these systems in areas ranging from biology to microelectronics. He has opened broad new technological avenues and has impacted human health in significant ways. Whitesides's research in materials chemistry has become an essential part of materials synthesis programs around the world.

Among Whitesides's many innovations are the synthesis and molecular organization of new classes of materials, pioneering self-assembled monolayers and microfluidic systems to enable the development of new drugs and extend soft materials into the world of three-dimensional microelectronics, and into consumer devices such as solar cells. Within this work he developed soft lithography, a set of methods for printing and molding organic-based substances to make complex patterns at the micron and nanometer level.

Whitesides combined these approaches for creating materials with the concept of polyvalency and developed a new paradigm for drug design. This has resulted in affordable medical diagnostics expected to have a major impact on health in the third world as well as new drugs that manage cholesterol, improve dialysis, and combat multiple drug-resistant pathogens.

Whitesides has received many awards. Among those from the American Chemical Society are the Award in Pure Chemistry, the Arthur C. Cope Award, and the Society's highest award, the Priestley Medal. From other institutions his awards include the Materials Research Society's Von Hippel Award, the Welch Award from the Welch Foundation, the American Institute of Chemists Gold Medal, and the Franklin Foundation's Benjamin Franklin Medal in Chemistry. In 1998, he was awarded the National Medal of Science. International recognition includes the Kyoto Prize for Advanced Technology, the Paracelsus Prize of the Swiss Chemical Society, the UAA-Dhirumbhai Ambani Award of the National Academy of Science in India, and the Prince of Asturias Foundation's Award in Science and Technology.

In addition to his academic research, he has helped found 12 companies in biotechnology and materials science and holds more than 50 patents. He is a member of the board of directors of Theravance, Hughes Research Laboratories, Surface Logix, Nano-Terra, Arsenal Biomedical, Diagnostics for All, and Paper Diagnostics. 